Original Article

\title{
Chitotriosidase as biomarker for early stage amyotrophic lateral sclerosis:
}

\section{A multicenter study}

Petra Steinacker $\mathrm{PhD}^{1}$, Emily Feneberg $\mathrm{MD} \mathrm{PhD}^{2}$, Steffen Halbgebauer $\mathrm{PhD}^{1}$, Simon Witzel $\mathrm{MD}^{1}$, Federico Verde $\mathrm{MD}^{3,4}$, Patrick Oeckl PhD ${ }^{1}$, Philip Van Damme MD, $\mathrm{PhD}^{5,6}$, Nayana Gaur ${ }^{7}$, Elizabeth Gray $\mathrm{PhD}^{2}$, Julian Grosskreutz $\mathrm{MD}^{7}$, Claude G. Jardel PharmD PhD ${ }^{8}$, Mykyta Kachanov ${ }^{9}$ Jens Kuhle $\mathrm{MD}^{10}$, Foudil Lamari $\mathrm{MD}^{8}$, Aleksandra Maceski $\mathrm{PhD}^{10}$, Maria del Mar Amador $\mathrm{MD}^{11}$, Benjamin Mayer $\mathrm{PhD}^{12}$, Claudia Morelli $\mathrm{MD}^{3}$, Susanne Petri $\mathrm{MD}^{13}$, Koen Poesen PharmD ${ }^{14}$, PhD, Joost Raaphorst $\mathrm{MD}^{15}$, François Salachas $\mathrm{MD}^{11}$, Vincenzo Silani $\mathrm{MD}^{3,4}$, Martin R. Turner $\mathrm{PhD}^{2}$, Marcel M. Verbeek PhD MSc${ }^{16}$, Alexander E. Volk MD ${ }^{9}$, Jochen H. Weishaupt MD ${ }^{1}$, Patrick Weydt MD ${ }^{17}$, Albert C. Ludolph MD ${ }^{1} \&$ Markus Otto MD ${ }^{1}$

1 Department of Neurology, Ulm University, Ulm, Germany

2 Nuffield Department of Clinical Neurosciences, University of Oxford, Oxford, UK

3 Department of Neurology - Stroke Unit and Laboratory of Neuroscience, Istituto Auxologico Italiano, IRCCS, Milan, Italy

4 Department of Pathophysiology and Transplantation - "Dino Ferrari" Center, Università degli Studi di Milano, Milan, Italy

5 University Hospitals Leuven, Department of Neurology, Leuven, Belgium

6 KU Leuven - University of Leuven, Department of Neurosciences, VIB - Center for Brain \& Disease Research, Experimental Neurology - Laboratory of Neurobiology, Leuven, Belgium

7 Department of Neurology, Jena University Hospital, Jena, Germany

8 Department of Metabolic Biochemistry, Hôpitaux Universitaires Pitié Salpeêtrière-Charles Foix, Paris, France

9 Institute of Human Genetics, University Medical Center Hamburg-Eppendorf, Hamburg, Germany

10 Neurology, Departments of Biomedicine, Medicine and Clinical Research, University and University Hospital Basel, Basel, Switzerland

11 Paris ALS Reference Center, Neurological Diseases Department, Hôpitaux Universitaires Pitié Salpeêtrière-Charles Foix, Paris, France

12 Institute for Epidemiology and Medical Biometry, Ulm University, Ulm, Germany

13 Department of Neurology, Hannover Medical School, Hannover, Germany;

14 Laboratory of molecular neurobiomarker research, Leuven Brain Institute, KU Leuven and Laboratory Medicine, University Hospitals of Leuven, Leuven, Belgium

15 Department of Neurology, Amsterdam Neuroscience Institute, Amsterdam UMC, University of Amsterdam, Amsterdam, The Netherlands 
16 Radboud University Medical Center, Donders Institute for Brain, Cognition and Behaviour, Departments of Neurology and Laboratory Medicine, Nijmegen, The Netherlands

17 Department for Neurodegenerative Disorders and Gerontopsychiatry, Bonn University, Bonn, Germany

\section{Corresponding author}

Prof. Dr. med. Markus Otto

Ulm University Hospital, Department of Neurology

Oberer Eselsberg 45, D-89081 Ulm

Phone +49-731-500-63010

Fax: +49-731-500-63012

markus.otto@uni-ulm.de

\section{Authors' email addresses}

petra.steinacker@uni-ulm.de, emily.feneberg@ndcn.ox.ac.uk, steffen.halbgebauer@uniulm.de, simon.witzel@uni-ulm.de, fdrc.verde@gmail.com, patrick.oeckl@uni-ulm.de, philip.vandamme@uzleuven.be,nayana.gaur@med.uni-jena.de, elizabeth.gray@ndcn.ox.ac.uk, julian.grosskreutz@med.uni-jena.de, claude.g.jardel@wanadoo.fr, mykyta.kachanov@stud.uke.uni-hamburg.de, jens.kuhle@usb.ch, foudil.lamari@aphp.fr, aleksandra.maceski@usb.ch, mariadelmar.amador@aphp.fr, benjamin.mayer@uni-ulm.de,c.morelli@auxologico.it, petri.susanne@mh-hannover.de, koen.poesen@uzleuven.be,j.raaphorst@amsterdamumc.nl, francois.salachas@aphp.fr, vincenzo@silani.com, martin.turner@ndcn.ox.ac.uk, marcel.verbeek@radboudumc.nl, a.volk@uke.de,jochen.weishaupt@uni-ulm.de, patrick.weydt@ukbonn.de, albert.ludolph@rku.de, markus.otto@uni-ulm.de

Words abstract: 249

Words main text: 2996

Figures: 4

Tables: 2

References: 36

\section{Supplementary data:}

Table S1: Summary of multivariate analysis results for CSF CHIT1, NfL, and pNfH levels in the prognosis of survival time and progression at follow-up. 


\section{ABSTRACT}

Objective: Levels of chitotriosidase (CHIT1) are increased in the cerebrospinal fluid (CSF) of amyotrophic lateral sclerosis (ALS) patients reflecting microglial activation. Here, we determine the diagnostic and prognostic potential of CHIT1 for early symptomatic ALS.

Methods: Overall, 275 patients from 8 European neurological centers were examined. We included ALS with $<6$ and $>6$ months from symptom onset, other motoneuron diseases (oMND), ALS mimics (DCon) and non-neurodegenerative controls (Con). CSF CHIT1 levels were analyzed for diagnostic power and association with progression and survival in comparison to the benchmark neurofilament. The 24-bp duplication polymorphism of CHIT1 was analyzed in a subset of patients $(\mathrm{N}=65)$.

Results: Homozygous CHIT1 duplication mutation carriers (9\%) invariably had undetectable CSF CHIT1 levels, while heterozygous carriers had similar levels as patients with wildtype CHIT1 ( $\mathrm{p}=0.414)$. In both early and late symptomatic ALS CHIT1 levels was increased, did not correlate with patients' progression rates, and was higher in patients diagnosed with higher diagnostic certainty. Neurofilament levels correlated with CHIT1 levels and prevailed over CHIT1 regarding diagnostic performance. Both CHIT1 and neurofilaments were identified as independent predictors of survival in late but not early symptomatic ALS. Evidence is provided that CHIT1 predicts progression in El Escorial diagnostic category in the group of ALS cases with a short duration.

Conclusions: CSF CHIT1 level may have additional value in the prognostication of ALS patients with a short history of symptoms classified in diagnostic categories of lower clinical certainty. To fully interpret apparently low CHIT1 levels knowledge of CHIT1 genotype is needed.

\section{Key words:}

amyotrophic lateral sclerosis, prognostic biomarker, chitotriosidase, neurofilaments 


\section{INTRODUCTION}

The diagnosis of ALS is currently based on clinical findings specified in the revised El Escorial criteria.(1) The median time from symptom onset to diagnosis is 14 months.(2) This is reduced by large in registers with a high capture-recapture rate,(3) however, early diagnosis of ALS is challenging because the diagnostic certainty relies on the regional extent of clinical and electrophysiological signs and furthermore, some other diseases can mimic ALS at an early stage. $(4,5)$

Neurochemical biomarkers are currently not included in the diagnostic criteria of ALS, but cerebrospinal fluid (CSF) biomarkers could aid early diagnosis and predict prognosis. Recently, chitinase 1 (chitotriosidase, CHIT1) was proposed as CSF marker of microglia activation in ALS.(6-9) CHIT1, the most abundant human chitinase and is expressed by cells of the monocyte/macrophage.(10) In single center studies increased CHIT1 protein levels in the CSF were shown to allow for the discrimination from healthy control subjects, patients with other neurodegenerative diseases and diseases mimicking ALS. Furthermore, CHIT1 level correlated with ALS disease progression and was associated with survival time.(6-9, 11-13) Recent studies suggest that CHIT1 rises around symptom onset pointing to the suitability as early biomarker for ALS. $(8,14)$

The aim of the present study was to examine whether the differential diagnostic appropriateness of CHIT1 can be confirmed for early and uncertain ALS cases in a multicentric approach. Furthermore, we aimed to examine whether CHIT1 could inform ALS prognosis, especially in early ALS. Data were analyzed in comparison to CSF neurofilaments as benchmark for neuronal affection and prognostic marker in ALS.(15-17)

\section{METHODS}




\section{Participants}

Overall, the study included 275 patients who were seen in specialized Neurological University Centers in Germany (Ulm, N=145; Hanover, $\mathrm{N}=18$; Jena, $\mathrm{N}=24$ ), Belgium (Leuven, $\mathrm{N}=13$ ), UK (Oxford, $\mathrm{N}=11$ ), France (Paris, $\mathrm{N}=21$ ), Italy (Milan, $\mathrm{N}=21$ ), and The Netherlands (Nijmegen, $\mathrm{N}=22$ ). The study was conducted according to the principles expressed in the Declaration of Helsinki. Collection and analysis of samples were approved by the local Ethics Committees. All patients gave written informed consent to their participation in the study.

Within our cohort, 161 patients were diagnosed with definite, probable, clinically probable laboratory supported, and possible ALS.(1) Patients presenting with isolated lower motor neuron signs were included as suspected ALS.

Patients were grouped in early (<6months) and late (>6months) symptomatic ALS.(15) The revised ALS functional rating scale (ALSFRS-R) was used to quantify the severity of physical symptoms.(18) Disease progression was expressed as decrease in the ALSFRS-R score per month (disease progression rate, $\mathrm{PR}$ ).

As disease comparison group we included 30 patients with other motoneuron diseases (oMND: $\mathrm{N}=16$ primary lateral sclerosis (PLS); $\mathrm{N}=9$ spinal muscular atrophy, $\mathrm{N}=5$ spinal and bulbar muscular atrophy, and furthermore 41 patients with ALS mimicking diseases (disease controls, DCon). The ALS mimic diagnoses were as follows: $\mathrm{N}=12$ polyneuropathy, $\mathrm{N}=8$ peripheral mononeuropathy, $\mathrm{N}=2$ brachial plexopathy, $\mathrm{N}=2$ radiculopathy, $\mathrm{N}=2$ myopathy, $\mathrm{N}=1$ juvenile parkinsonism, $\mathrm{N}=1$ lipid storage disease, $\mathrm{N}=3$ spastic paraplegia, $\mathrm{N}=3$ fasciculation syndromes, $\mathrm{N}=1$ cerebrovascular disease, $\mathrm{N}=2$ myelopathy, $\mathrm{N}=4$ no neurological diagnosis. A group of 43 participants who had no signs of neurodegeneration or acute or chronic inflammation of the brain served as control group (Con). 
Genetic testing of 19 ALS cases from Ulm identified 2 SOD1 and 4 C9orf72 mutation carriers.

For 65 patients from Ulm the CHIT1 24-bp duplication polymorphism (c.1049_1072dup, NM_003465.2) was determined.(7, 19)

Demographic and disease characteristics of the patient cohort which is partially identical to cohorts we reported on in previous studies $(15,17,20)$ are given in Table 1.

\section{Laboratory markers}

CSF was centrifuged, aliquoted in polypropylene tubes and stored within two hours at $-80^{\circ} \mathrm{C}$ until analysis. Samples were measured blinded to any clinical data. Commercially available ELISA kits were used for CHIT1 (MBL, Diegem, Belgium) with a limit of detection of 48 $\mathrm{pg} / \mathrm{mL}$. CSF was prediluted 1:5 to 1:50 and the limit of quantitation (LOQ was set to $281 \mathrm{pg} / \mathrm{mL}$. NfL (IBL, Hamburg, Germany) and pNfH (Biovendor, Heidelberg, Germany) were measured according to the manufacturers' instructions.(17) Except for samples from Ulm, only one NfL measurement could be performed due to lack of sample with an upper LOQ of $10000 \mathrm{pg} / \mathrm{mL}$. The mean inter-assay CV was $<20 \%$ for the CHIT1, NfL, and pNfH ELISAs.

\section{Statistical analyses}

Two-tailed unpaired Mann-Whitney U-test and Kruskal-Wallis test with Dunn's post-hoc test were used to determine differences between groups. The optimal cut-off level for dichotomising values was selected as the one resulting in the highest Youden index. The receiver operating characteristics (ROC) curve was used for a graphical visualization of the impact of the variation in the cut-off values. Correlation was calculated using Spearman's rank correlation test. Cox regression analysis was used to determine the prognostic value considering covariates identified by univariate analyses. Hazard ratios are illustrated by Kaplan-Meier plots showing curves for biomarker tertile levels. Statistical analysis was performed using GraphPadPrism7.0 and SPSS24. 
Table 1: Demographic characteristics, CHIT1 genotypes, and biomarker concentrations for the multicenter cohort.

\begin{tabular}{|c|c|c|c|c|c|c|}
\hline & ALS & early ALS & late ALS & oMND & DCon & Con \\
\hline $\mathbf{N}$ & 161 & 58 & 103 & 30 & 42 & 43 \\
\hline gender (\% female) & 36 & 34 & 37 & 27 & 29 & 65 \\
\hline age (years) & $62(34-85)$ & $62(35-84)$ & $61(34-85)$ & $60(23-82)$ & $56(26-79)$ & $51(33-80)$ \\
\hline $\begin{array}{l}\text { site of onset } \\
(\text { spinal/bulbar })^{\mathrm{a}}\end{array}$ & $60 / 92$ & $21 / 34$ & $39 / 58$ & & & \\
\hline disease duration (months) & $10.3(1-78)$ & $4.6(1-6)$ & $13(6.3-78)$ & & & \\
\hline ALSFRS-R & $43(6-48)$ & $44(6-48)$ & $42(23-48)$ & & & \\
\hline progression rate & $0.48(0-8.7)$ & $1(0-8.7)$ & $0.39(0-2.8)$ & & & \\
\hline $\begin{array}{l}\text { clinical certainty at } \\
\text { baseline }^{\text {b }}\end{array}$ & $\begin{array}{c}13 \text { definite } \\
49 \text { probable } \\
45 \text { probable lab } \\
23 \text { possible } \\
22 \text { suspected }\end{array}$ & $\begin{array}{c}5 \text { definite } \\
26 \text { probable } \\
11 \text { probable lab } \\
9 \text { possible } \\
7 \text { suspected }\end{array}$ & $\begin{array}{c}8 \text { definite } \\
23 \text { probable } \\
34 \text { probable lab } \\
14 \text { possible } \\
15 \text { suspected }\end{array}$ & & & \\
\hline $\begin{array}{l}\text { CHIT1 pm (homo- } \\
\text { /heterozygous/WT) }\end{array}$ & $4 / 15 / 26$ & $3 / 7 / 8$ & $1 / 8 / 18$ & $1 / 3 / 6$ & $1 / 1 / 8$ & \\
\hline CHIT1 in $\operatorname{CSF}^{\mathrm{c}}(\mathrm{pg} / \mathrm{mL})$ & $\begin{array}{c}7519(335- \\
196160)\end{array}$ & $\begin{array}{c}10412(1385- \\
196150)\end{array}$ & $6370(335-97150)$ & $2659(283-14655)$ & $\begin{array}{c}3210(380- \\
74600)\end{array}$ & $\begin{array}{c}2060(560- \\
55649)\end{array}$ \\
\hline NfL in CSF (pg/mL) & $5606(191-25650)$ & $6802(191-25650)$ & $5100(407-24240)$ & $978(100-8557)$ & $740(178-5240)$ & $1225(166-4758$ \\
\hline pNfH in CSF $(p g / m L)$ & $2706(171-13260)$ & $3819(319-12670)$ & $1983(171-13620)$ & $318(62-6195)$ & $352(62-4160)$ & $276(62-1203)$ \\
\hline
\end{tabular}

Data are given as median and range.

a: not recorded for 9 patients

b: not recorded for 11 patients

c: excluding 24 samples with CHIT1 below LLOQ 


\section{RESULTS}

\section{Characteristics of the study cohort}

Demographic data, disease characteristics, and biomarker concentrations are summarized in

\section{Table 1.}

Con and DCon patients were younger than ALS and oMND patients $(\mathrm{p}<0.05)$ and the Con group comprised a larger proportion of women than the other groups (Chi-square $=15.19$, $\mathrm{df}=1, \mathrm{p}=0.022$ ). Early symptomatic ALS patients were less affected $(\mathrm{p}=0.0095)$ as quantified by means of the ALSFRS-R score and had a faster disease progression $(\mathrm{p}<0.0001)$ compared to later cases, while site of onset was equally distributed (Chi-square 0.0602, $\mathrm{df}=1$, $\mathrm{p}=0.8062) . \mathrm{N}=123$ ALS patients (64\% of early $82 \%$ of late ALS) received a follow-up examination. Among these, 46 ALS patients (29\% of the early and $28 \%$ of the late cases) progressed resulting in an increase of the clinical category/diagnostic certainty.

\section{Frequency of CHIT1 24-bp duplication polymorphism}

Frequencies of patients carrying the CHIT1 24-bp duplication polymorphism are shown in Fig. 1A. Patients who are homozygous for the polymorphism were found in every diagnostic group. Statistically, there was no difference in the genotype frequencies in ALS compared to oMND and DCon patients (Chi-square=1.197, $\mathrm{df}=2, \mathrm{p}=0.5497$ ).

\section{CHIT1 protein levels in the diagnostic groups and dependent on the CHIT1 genotype}

All homozygous duplication carriers had CHIT1 levels below the LLOQ, while heterozygous and WT carriers invariably had quantifiable CHIT1 in the CSF. CHIT1 level was similar in heterozygous compared to WT carriers for all ALS ( $p=0.1737$, Fig. 1B) and early ALS ( $p=0.7789$ ) but reduced in late ALS ( $p=0.0187$, Fig. 1C). Overall, there was no 
difference in CHIT1 levels of WT and heterozygous carriers in all diagnostic groups $(\mathrm{p}=0.2135)$ or in the early and late symptomatic ALS patients $(\mathrm{p}=0.1893)$.

Early and late symptomatic ALS patients had similar CHIT1 levels $(\mathrm{p}=0.0667)$ and both could be discriminated from Con, DCon, and oMND with slightly better results for early diagnosed ALS (Fig. 2A). In the DCon group there were some patients with relatively high CHIT1 levels, especially a patient with radiculopathy $(17660 \mathrm{pg} / \mathrm{mL})$ and a patient with benign fasciculation syndrome $(14130 \mathrm{pg} / \mathrm{mL})$. Looking at the oMND patients we found lower CHIT1 levels in SMA/SBMA (2757 $\pm 1455 \mathrm{pg} / \mathrm{mL})$ compared to PLS (5736 \pm 4476 $\mathrm{pg} / \mathrm{mL})$.

Early ALS could be discriminated from Con with a sensitivity of 55\% and a specificity of $89 \%$ and from DCon with $82 \%$ sensitivity and $84 \%$ specificity. For late ALS we calculated $88 \%$ sensitivity and $40 \%$ specificity for the discrimination from Con and $79 \%$ sensitivity and $81 \%$ specificity for the discrimination from DCon (Fig. 2B).

\section{Comparison of the diagnostic performance of CHIT1 and neurofilaments}

Neurofilament levels, which did not differ dependent on the CHIT1 genotype (data not shown), were higher in the early compared to the late symptomatic ALS subgroups (Fig. 2C,E). ROC analysis yielded higher AUC values for NfL and pNfH (Fig. 2D,F) than for CHIT1 resulting in better sensitivities and specificities as given beside the respective graphs.

\section{Correlation of CHIT1 with demographic and disease parameters, neurofilaments and} patients' survival

Analysis of potential associations of the CHIT1 levels with the ALS patients' demographic and disease characteristics as well as with their NfL and pNfH CSF levels revealed a robust correlation of with neurofilaments only, observed for both the early and late symptomatic ALS subgroups. 
There was a weak correlation of CHIT1 level with the ALS patients' age, age at disease onset, and PR. A summary of correlation analyses results is given in Table 2.

Table 2: Spearman correlation coefficients and p-values for the association of CHIT1 with demographic, disease and biochemical parameters.

\begin{tabular}{|c|c|c|c|c|}
\hline & & all ALS & ALS $<6 \mathrm{mo}$ & $\mathrm{ALS}>\mathbf{6 m o}$ \\
\hline \multirow{3}{*}{ Age } & rho & -0.191 & -0.316 & -0.118 \\
\hline & $95 \% C I$ & -0.346 to -0.026 & -0.550 to -0.036 & -0.316 to 0.089 \\
\hline & $p$ & 0.02 & 0.0238 & 0.2485 \\
\hline \multirow{3}{*}{ Age at onset } & rho & -0.180 & -0.340 & -0.093 \\
\hline & $95 \% C I$ & -0.337 to -0.013 & -0.573 to -0.056 & -0.292 to 0.115 \\
\hline & $p$ & 0.0302 & 0.0169 & 0.3671 \\
\hline \multirow{3}{*}{ Disease duration } & rho & -0.177 & -0.158 & -0.077 \\
\hline & $95 \% C I$ & -0.333 to -0.012 & -0.422 to 0.132 & -0.278 to 0.130 \\
\hline & $p$ & 0.0313 & 0.2694 & 0.4547 \\
\hline \multirow{3}{*}{ Progression rate } & rho & 0.209 & 0.100 & 0.158 \\
\hline & $95 \% C I$ & 0.039 to 0.368 & -0.204 to 0.387 & -0.055 to 0.356 \\
\hline & $p$ & 0.0138 & 0.5071 & 0.1336 \\
\hline \multirow{3}{*}{ ALSFRS-R } & rho & -0.050 & -0.043 & -0.123 \\
\hline & $95 \% C I$ & -0.220 to 0.123 & -0.337 to 0.258 & -0.325 to 0.090 \\
\hline & $p$ & 0.5632 & 0.7744 & 0.2429 \\
\hline \multirow{3}{*}{ Survival } & rho & 0.099 & 0.363 & 0.022 \\
\hline & $95 \% C I$ & -0.190 to 0.372 & -0.253 to 0.769 & -0.309 to 0.348 \\
\hline & $p$ & 0.4905 & 0.224 & 0.8946 \\
\hline \multirow{3}{*}{ NfL } & rho & 0.517 & 0.478 & 0.505 \\
\hline & $95 \% C I$ & 0.383 to 0.629 & 0.226 to 0.671 & 0.335 to 0.644 \\
\hline & $p$ & $<0.0001$ & 0.0004 & $<0.0001$ \\
\hline \multirow{3}{*}{ pNfH } & rho & 0.572 & 0.499 & 0.594 \\
\hline & $95 \% C I$ & 0.448 to 0.674 & 0.251 to 0.685 & 0.443 to 0.712 \\
\hline & $p$ & $<0.0001$ & 0.0002 & $<0.0001$ \\
\hline
\end{tabular}

\section{CHIT1 levels in ALS diagnostic categories}

At first examination, CHIT1 levels differed in each clinical ALS category compared to Con ( $\mathrm{p}<0.0001$ for definite, probable, and laboratory-supported probable, $\mathrm{p}<0.001$ for possible and suspected ALS) and for the more certain categories compared to the DCon levels ( $\mathrm{p}<0.0001$ for probable and $\mathrm{p}<0.05$ for definite ALS). Within the group of ALS patients a 
trend for increasing levels with increasing diagnostic certainty was observed (Fig. 3A). Also, for the single diagnostic categories we found comparable levels in stable and progressing ALS patients with the exception that those patients who progressed from suspected ALS to either possible, laboratory-supported, probable or definite ALS had higher CHIT1 levels than patients who still had suspected ALS at follow-up (p=0.0121, Fig. 3B).

\section{CHIT1 in the prognosis of progression at follow-up and survival}

Applying univariate Cox regression analysis effects on CHIT1 levels were determined for the patients' age (HR: 10.4, 95\% CI: 4.4-33.5, $\mathrm{p}<0.0001$ for patients at age $>70$ years compared to $<40$ years), the PR (HR: $2.4,95 \%$ CI: $1.2-4.8, p=0.016$ for high versus low PR tertile) and for the site of onset (higher in spinal onset, HR: 3.3, 95\% CI: 1.4-7.8, p=0.007). The 6 enrolled fALS patients had shorter survival times (HR: 3.3, 95\% CI: 1.4-7.8, p=0.007) and therefore were excluded from analysis.

CHIT1 could be identified as independent predictor of survival $(\mathrm{p}=0.007)$ with a HR of 3.8 (95\% CI: 1.2-12.0, $\mathrm{p}=0.021)$ for ALS patients with upper compared to low tertile CHIT1 ($2 \log$-likelihood $(-2 \mathrm{LL})=200.8$, Chi-square 43.5, $\mathrm{df}=1, \mathrm{p}<0.0001$, Fig. 4A). This result could be confirmed for late ALS patients $(\mathrm{p}=0.041)$ and by tendency also for early ALS patients $(p=0.07$, Fig. 4B,C).

Next, we analyzed if CHIT1 levels at baseline examination can predict change at follow-up towards a higher El Escorial category. For this we compared patients who stayed stable at follow-up with those patients who progressed. Self-explaining, definite ALS cases had to be excluded here. As follow-up information was available for patients from all involved centers with evidence for shorter times to follow-up for the multicenter cohort patients compared to the Ulm cohort patients (log-rank Chi-square 2.92, $\mathrm{df}=1, \mathrm{p}=0.088$ ), a potential center effect was regarded as covariate in Cox regression. Furthermore, the initial El Escorial stadium was regarded as confounder. Cox regression with the factors CHIT1, age, center, and initial 
diagnostic category $(-2 \mathrm{LL}=225.2$, Chi-square $29.9, \mathrm{df}=6, \mathrm{p}<0.0001)$ revealed no prognostic value of CHIT1 for all ALS patients ( $\mathrm{p}=0.128$, Fig. 4D) and late ALS patients ( $\mathrm{p}=0.359$, Fig. 4F), whereas the early diagnosed ALS patients with highest tertile CHIT1 levels had a higher risk for progression at follow-up than early ALS patients with CHIT1 levels in the lowest tertile $(\mathrm{HR}=13.4,95 \% \mathrm{CI}: 1.3-135.8, \mathrm{p}=0.028$, Fig. 4E).

A summary of Cox proportional hazard analysis results for CHIT1 in the prognosis of progression and survival is given in Supplementary Table S1.

\section{Neurofilaments' prognostic properties}

ALS patients with high tertile NfL or pNfH had 5.3 times (95\% CI: $1.8-11.9, \mathrm{p}=0.001)$ and 3.0 times (95\% CI: 1.2-7.6, $\mathrm{p}=0.022$ ) higher risk to die within the observation period compared to patients with low tertile $\mathrm{NfL}$ or $\mathrm{pNfH}$, respectively, when using the same parameters as covariates that were used for analysis of CHIT1. For late ALS, these results were confirmed for NfL (HR 5.9, 95\% CI: 1.7-20.6, $\mathrm{p}=0.005$ ) and a trend could be seen for pNfH $(\mathrm{HR}=3.0,95 \%$ CI: 0.9-9.5, $\mathrm{p}=0.066)$.

With regards to prognosis of progression at follow-up by means of transition to a diagnostic category of higher certainty we saw for NfL, but not $\mathrm{pNfH}$, a trend for a contribution in the model $(\mathrm{p}=0.069$ and $\mathrm{p}=0.353)$.

By tertile analysis we did not obtain significant results for $\mathrm{Nf}$ when looking at all ALS cases together or at the late ALS subgroup. Early ALS patients with low NfL levels had the lowest risk for progression at follow-up and patients with middle tertile NfL levels had the highest risk (HR 26.8, 95\% CI: 1.2-598, $\mathrm{p}=0.038$ ). The level of $\mathrm{pNfH}$ was not a predictor of progression in early ALS patients $(\mathrm{p}=0.414)$.

A summary of Cox proportional hazard analysis results for $\mathrm{NfL}$ and $\mathrm{pNfH}$ in the prognosis of progression and survival is given in Supplementary Table S1. 


\section{DISCUSSION}

There is a need for biomarkers securing the diagnosis of ALS at early stages and informing prognosis in order to reduce the diagnostic delay and to stratify patients for upcoming therapies.(21) With the present study we aimed to assess the suitability of CHIT1 as marker for an ALS diagnosis within six months after symptom onset and for prognosis of progression and survival. We analyzed for a subset of patients the common CHIT1 24-bp duplication polymorphism and its effect on CSF CHIT1 level. Furthermore, we compared the significance of the inflammation marker CHIT1 with that of the neurodegeneration markers neurofilaments.

Of the genotyped patients, 9\% carried the homozygous 24-bp duplication of CHIT1 independent of the diagnosis which is in good accordance with frequency data of previous reports.(7, 19, 22-24) In our sample, homozygous CHIT1 polymorphism was invariantly associated with CHIT1 levels below the quantitation threshold, in line with reports on lower levels in carriers(19) and corresponding to complete enzyme activity deficiency,(19, 23, 25, 26) but heterozygous and WT carriers had similar levels, in contrast to published observations. $(7,27)$ Previous studies described that heterozygous carriers have half of the activity level of CHIT1 in serum(25) which, based on our data, cannot be assigned solely to the protein levels. Further analyses should focus on the influence of the heterozygous CHIT1 duplication mutation on CHIT1 protein and activity levels and determine the CHIT1 diagnostic and prognostic properties for ALS.

The CHIT1 levels determined in this multicenter study were in the same range as in previous single center studies utilizing the same ELISA. $(6,9,12)$ For the differentiation of ALS and ALS mimics, we obtained weaker sensitivities and specificities compared to our previous 
study(8) and here also the largest differences between the centers involved here as well as between our and published data $(6,11)$ were observed. This could be ascribed to differences in the cohort composition, i.e. emphasis on early symptom ALS. Also, there was a high variety in CHIT1 in the DCo group. This group potentially includes patients with diseases characterized by macrophage activation and increased CHIT1 in the periphery.(10) The effect of blood CHIT1 on the levels of CHIT1 in CSF was not examined but should be done in the future.

In the oMND group CHIT1 levels were relatively high due to high levels in some of the PLS patients who were included to be consistent with our multicenter study of $\mathrm{Nf}$ in early stage ALS.(15) Increased CHIT1 levels in PLS compared to healthy controls were observed in a recently published pilot study.(28) Furthermore, it has been shown that microglia activation correlated with UMN clinical symptoms(29) and CSF CHIT1 levels with the sum of regions affected by UMN and LMN degeneration in ALS.(6) Our finding could speak for CHIT1 as UMN degeneration marker and might be judged as supporting the hypothesis of PLS being a variant of ALS.(30)

While CHIT1 correlated strongly with NfL and pNfH it did not outperform neurofilaments diagnostic performance, in accordance with published data. $(6-9,11)$ For the prognosis of the clinical course of ALS and prediction of survival times, however, CHIT1 performed remarkably well. We found a trend for increasing CHIT1 levels with increasing clinical diagnostic certainty. Of note, neurofilament CSF levels were found to be similarly increased across diagnostic categories.(15)

It is important to emphasize that the applied El Escorial criteria(1) do not represent a staging scheme for ALS. The clinical categories can be prone to bias, e.g. the category of clinically probable-laboratory supported ALS could be skewed towards slow progressors with a longer diagnostic delay.(31) However, as the categories describe the certainty that the phenotype is 
ALS, the categories refer to the severity of pathology involved.(32) Additional insights might be gained by analysis of potential correlation of CHIT1 levels with ALS clinical staging scales as recently standardized operation procedures are available enabling for implementation especially in multicenter studies. $(33,34)$

CHIT1 levels were not robustly associated with the disease progression rate in contrast to previous studies. $(8,9,11,13,35)$ This could be due to the large number of early symptomatic cases and a potential imprecision of the PRs which is supported by the less robust correlation of neurofilament levels with the progression rate (15) compared to other studies $(17,21)$ besides a correlation with CHIT1, as expected.(8) To account for an influence by PR and further potential covariates we conducted multivariate regression analyses. Here, CHIT1 was superior to neurofilaments in the prediction of disease progression. Similar analyses were recently performed regarding covariates identified before to influence prognostic conclusiveness $(6,36)$ showing that CHIT1, although correlating with survival time, is not an independent predictor of survival. Future studies have to clarify if it is possible to make a point about progression or survival time in ALS or ALS subgroups when specific covariates are taken into account.

An important limitation of our study is that we were not able to genotype CHITI for every patient. Furthermore, there might be a selection bias for the Centers as diagnostic subgroups were not matched with regard to patients' numbers and diagnostic category. Finally, within the group of early ALS the average PR was higher than in the late ALS group. Although we included the PR as covariate in some analyses a bias cannot be completely excluded and future studies should aim for harmonization of PRs in ALS subgroups.

Taken together we confirmed the diagnostic value of CHIT1 for ALS in a multicentric cohort of patients and extended the proof to early symptomatic ALS stages. We conclude that elevated CHIT1 CSF levels in suspected ALS support the diagnosis, especially when the 
patients' symptoms are present for less than six months at sampling, and that CHIT1 complement the validity of neurofilaments. Furthermore, as CHIT1 could predict ALS patients' progress at follow-up, we propose CHIT1 as stratification biomarker for clinical trials.

\section{Study funding:}

This study was supported by the EU Joint Programme - Neurodegenerative Diseases (JPND) research networks under Grants Prefrontals (01ED1512), GENF-Prox

(01ED2008A), and ONWebDUALS; the German Federal Ministry of Education and Research under Grant (FTLDc O1GI1007A); the foundation of the state BadenWürttemberg under Grant D.3830; the Thierry Latran foundation; the ALS Association; Boehringer Ingelheim Ulm University BioCenter under Grant D.5009; a TBM grant from FWO-Vlaanderen ( ${ }^{\circ}$ T003519N); by the German Society for Muscle Diseases (DGM); the German Neuromuscular Society; the German Israeli Foundation (GIF) for Scientific Research and Development.

PVD holds a senior clinical investigatorship of FWO-Vlaanderen and is supported by E. von Behring Chair for Neuromuscular and Neurodegenerative Disorders, the ALS Liga België and the KU Leuven funds "Een Hart voor ALS", "Laeversfonds voor ALS Onderzoek" and the "Valéry Perrier Race against ALS Fund". Several authors of this publication are member of the European Reference Network for Rare Neuromuscular Diseases (ERN-NMD). VS receives or has received research supports from the Italian Ministry of Health, AriSLA (Fondazione Italiana di Ricerca per la SLA), and E-Rare Joint Transnational Call. MRT is funded by the Motor Neurone Disease Association. 


\section{Disclosures:}

The author(s) declared the following potential conflicts of interest with respect to the research, authorship, and/or publication of this article:

PS, EF, SH, SW, FV, PO, NG, EG, JG, CJ, MK, JK, FL, AM, MDMA, BM, CM, KP, JR, FS, MMV, AEV, JHW, PW, ACL, and MO report no disclosures.

Prof. Van Damme has served on advisory board meetings for Biogen, Pfizer, Alexion Therapeutics, Cytokinetics, CSL Behring.

Prof. Petri received speaking honoraria from Desitin Pharma, Biogen, Novartis and consulting fees from Cytokinetics Inc., Roche and Biogen.

Prof. Silani is in the Editorial Board of Amyotroph Lateral Sclerosis, European Neurology, American Journal of Neurodegenerative Diseases, Frontiers in Neurology; received compensation for consulting services and/or speaking activities from AveXis, Cytokinetics, Italfarmaco, and Zambon.

Prof. Turner received limited payment in kind (pNfH assay kits) from Euroimmun UK in 2018.

\section{Availability of data and material}

Any data not published within the article will be shared in pseudonymized form by request from any qualified investigator for purposes of replicating procedures.

\section{Acknowledgements:}

For their excellent technical assistance, we are grateful to Stephen Meier, Sandra Hübsch, Dagmar Schattauer, Alice Beer, and Mehtap Türedi.

We are deeply indebted to the patients for participating in this study. 


\section{REFERENCES}

(1) Brooks BR, Miller RG, Swash M, Munsat TL. El Escorial revisited: revised criteria for the diagnosis of amyotrophic lateral sclerosis. Amyotroph Lateral Scler Other Motor Neuron Disord 2000;1:293-299.

(2) Chio A. ISIS Survey: an international study on the diagnostic process and its implications in amyotrophic lateral sclerosis. J Neurol 1999;246 Suppl 3:III1-5.

(3) Rosenbohm A, Peter RS, Erhardt S, Lule D, Rothenbacher D, Ludolph AC, et al. Epidemiology of amyotrophic lateral sclerosis in Southern Germany. J Neurol 2017;264:749-757.

(4) Traynor BJ, Codd MB, Corr B, Forde C, Frost E, Hardiman O. Amyotrophic lateral sclerosis mimic syndromes: a population-based study. Arch Neurol 2000;57:109-113.

(5) Turner MR, Talbot K. Mimics and chameleons in motor neurone disease. Pract Neurol 2013;13:153-164.

(6) Gille B, De Schaepdryver M, Dedeene L, Goossens J, Claeys KG, Van Den Bosch L, et al. Inflammatory markers in cerebrospinal fluid: independent prognostic biomarkers in amyotrophic lateral sclerosis? J Neurol Neurosurg Psychiatry 2019;90:1338-1346.

(7) Oeckl P, Weydt P, Steinacker P, Anderl-Straub S, Nordin F, Volk AE, et al. Different neuroinflammatory profile in amyotrophic lateral sclerosis and frontotemporal dementia is linked to the clinical phase. J Neurol Neurosurg Psychiatry 2019;90:4-10.

(8) Steinacker P, Verde F, Fang L, Feneberg E, Oeckl P, Roeber S, et al. Chitotriosidase (CHIT1) is increased in microglia and macrophages in spinal cord of amyotrophic lateral sclerosis and cerebrospinal fluid levels correlate with disease severity and progression. J Neurol Neurosurg Psychiatry 2018;89:239-247. 
(9) Thompson AG, Gray E, Bampton A, Raciborska D, Talbot K, Turner MR. CSF chitinase proteins in amyotrophic lateral sclerosis. J Neurol Neurosurg Psychiatry 2019;90:12151220.

(10) Kanneganti M, Kamba A, Mizoguchi E. Role of chitotriosidase (chitinase 1) under normal and disease conditions. J Epithel Biol Pharmacol 2012;5:1-9.

(11) Thompson AG, Gray E, Thezenas ML, Charles PD, Evetts S, Hu MT, et al. Cerebrospinal fluid macrophage biomarkers in amyotrophic lateral sclerosis. Ann Neurol 2018;83:258-268.

(12) Varghese AM, Sharma A, Mishra P, Vijayalakshmi K, Harsha HC, Sathyaprabha TN, et al. Chitotriosidase - a putative biomarker for sporadic amyotrophic lateral sclerosis. Clin Proteomics 2013;10:19.

(13) Vu L, An J, Kovalik T, Gendron T, Petrucelli L, Bowser R. Cross-sectional and longitudinal measures of chitinase proteins in amyotrophic lateral sclerosis and expression of CHI3L1 in activated astrocytes. J Neurol Neurosurg Psychiatry 2020;91:350-358.

(14) Gray E, Thompson AG, Wuu J, Pelt J, Talbot K, Benatar M, et al. CSF chitinases before and after symptom onset in amyotrophic lateral sclerosis. Ann Clin Transl Neurol 2020.

(15) Feneberg E, Oeckl P, Steinacker P, Verde F, Barro C, Van Damme P, et al. Multicenter evaluation of neurofilaments in early symptom onset amyotrophic lateral sclerosis. Neurology 2018;90:e22-e30.

(16) Lu CH, Macdonald-Wallis C, Gray E, Pearce N, Petzold A, Norgren N, et al. Neurofilament light chain: A prognostic biomarker in amyotrophic lateral sclerosis. Neurology 2015;84:2247-2257.

(17) Steinacker P, Feneberg E, Weishaupt J, Brettschneider J, Tumani H, Andersen PM, et al. Neurofilaments in the diagnosis of motoneuron diseases: a prospective study on 455 patients. J Neurol Neurosurg Psychiatry 2016;87:12-20. 
(18) Cedarbaum JM, Stambler N, Malta E, Fuller C, Hilt D, Thurmond B, et al. The ALSFRS-R: a revised ALS functional rating scale that incorporates assessments of respiratory function. BDNF ALS Study Group (Phase III). J Neurol Sci 1999;169:13-21.

(19) Boot RG, Renkema GH, Verhoek M, Strijland A, Bliek J, de Meulemeester TM, et al. The human chitotriosidase gene. Nature of inherited enzyme deficiency. J Biol Chem $1998 ; 273: 25680-25685$.

(20) Verde F, Steinacker P, Weishaupt JH, Kassubek J, Oeckl P, Halbgebauer S, et al. Neurofilament light chain in serum for the diagnosis of amyotrophic lateral sclerosis. $\mathbf{J}$ Neurol Neurosurg Psychiatry 2019;90:157-164.

(21) Verde F, Silani V, Otto M. Neurochemical biomarkers in amyotrophic lateral sclerosis. Curr Opin Neurol 2019;32:747-757.

(22) Martinez-Merino L, Iridoy M, Galbete A, Roldan M, Rivero A, Acha B, et al. Evaluation of Chitotriosidase and CC-Chemokine Ligand 18 as Biomarkers of Microglia Activation in Amyotrophic Lateral Sclerosis. Neurodegener Dis 2018;18:208-215.

(23) Ries M, Schaefer E, Luhrs T, Mani L, Kuhn J, Vanier MT, et al. Critical assessment of chitotriosidase analysis in the rational laboratory diagnosis of children with Gaucher disease and Niemann-Pick disease type A/B and C. J Inherit Metab Dis 2006;29:647-652.

(24) Rodrigues MR, Sa Miranda MC, Amaral O. Allelic frequency determination of the 24bp chitotriosidase duplication in the Portuguese population by real-time PCR. Blood Cells Mol Dis 2004;33:362-364.

(25) Canudas J, Cenarro A, Civeira F, Garci-Otin AL, Aristegui R, Diaz C, et al. Chitotriosidase genotype and serum activity in subjects with combined hyperlipidemia: effect of the lipid-lowering agents, atorvastatin and bezafibrate. Metabolism 2001;50:447450. 
(26) Pagliardini V, Pagliardini S, Corrado L, Lucenti A, Panigati L, Bersano E, et al. Chitotriosidase and lysosomal enzymes as potential biomarkers of disease progression in amyotrophic lateral sclerosis: a survey clinic-based study. J Neurol Sci 2015;348:245-250. (27) Abu-Rumeileh S, Steinacker P, Polischi B, Mammana A, Bartoletti-Stella A, Oeckl P, et al. CSF biomarkers of neuroinflammation in distinct forms and subtypes of neurodegenerative dementia. Alzheimers Res Ther 2019;12:2.

(28) Verde F, Zaina G, Bodio C, Borghi MO, Soranna D, Peverelli S, et al. Cerebrospinal fluid phosphorylated neurofilament heavy chain and chitotriosidase in primary lateral sclerosis. J Neurol Neurosurg Psychiatry 2020.

(29) Brettschneider J, Toledo JB, Van Deerlin VM, Elman L, McCluskey L, Lee VM, et al. Microglial activation correlates with disease progression and upper motor neuron clinical symptoms in amyotrophic lateral sclerosis. PLoS One 2012;7:e39216.

(30) Ludolph A, Drory V, Hardiman O, Nakano I, Ravits J, Robberecht W, et al. A revision of the El Escorial criteria - 2015. Amyotroph Lateral Scler Frontotemporal Degener 2015;16:291-292.

(31) Braun N, Macklin EA, Sinani E, Sherman A, Weber M. The revised El Escorial criteria "clinically probable laboratory supported ALS"-once a promising now a superfluous category? Amyotroph Lateral Scler Frontotemporal Degener 2019:1-5.

(32) Al-Chalabi A, Hardiman O, Kiernan MC, Chio A, Rix-Brooks B, van den Berg LH. Amyotrophic lateral sclerosis: moving towards a new classification system. Lancet Neurol 2016;15:1182-1194.

(33) Roche JC, Rojas-Garcia R, Scott KM, Scotton W, Ellis CE, Burman R, et al. A proposed staging system for amyotrophic lateral sclerosis. Brain 2012;135:847-852.

(34) Balendra R, Al Khleifat A, Fang T, Al-Chalabi A. A standard operating procedure for King's ALS clinical staging. Amyotroph Lateral Scler Frontotemporal Degener 2019;20:159-164. 
(35) Gille B, De Schaepdryver M, Goossens J, Dedeene L, De Vocht J, Oldoni E, et al. Serum neurofilament light chain levels as a marker of upper motor neuron degeneration in patients with Amyotrophic Lateral Sclerosis. Neuropathol Appl Neurobiol 2019;45:291304.

(36) Westeneng HJ, Debray TPA, Visser AE, van Eijk RPA, Rooney JPK, Calvo A, et al. Prognosis for patients with amyotrophic lateral sclerosis: development and validation of a personalised prediction model. Lancet Neurol 2018;17:423-433. 


\section{FIGURE LEGENDS}
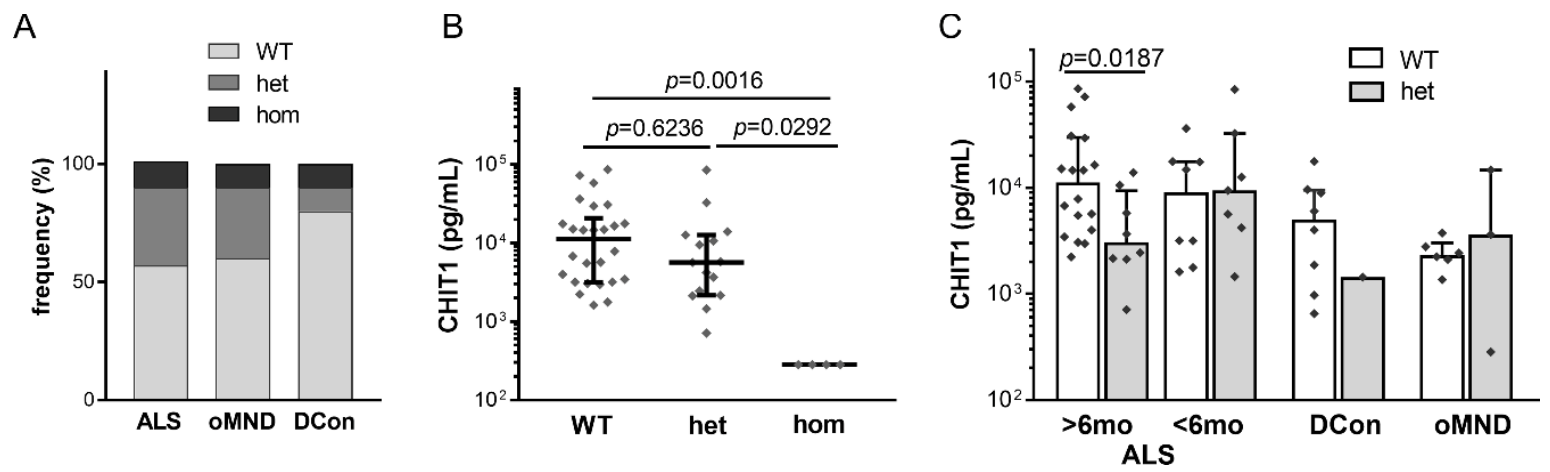

Figure 1: CSF CHIT1 levels dependent on CHIT1 genotype.

A: Frequency of the 24-bp duplication in CHITIfor the diagnostic groups. B: CSF CHIT1 levels in ALS patients depending on the CHIT1 polymorphism. Bars indicate median and IQR, the p-values result from Dunn's multiple comparison test. C: CHIT1 levels in patients without CHIT1 24-bp duplication compared to levels in heterozygous carriers for the diagnostic groups. Bars indicate mean levels with SD, Mann-Whitney $p$-values are given. WT, wildtype; het, heterozygous; hom, homozygous. 
A

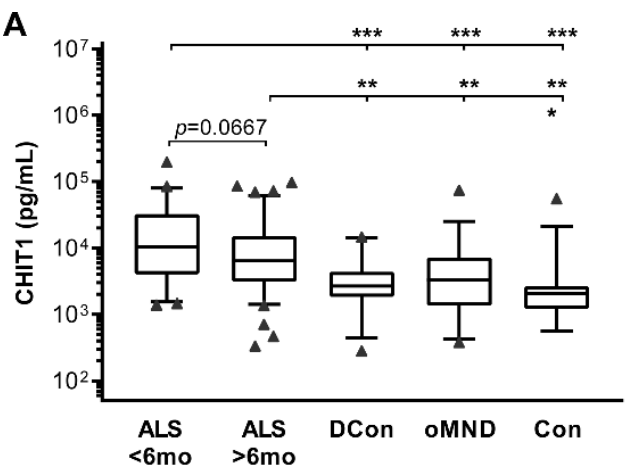

C

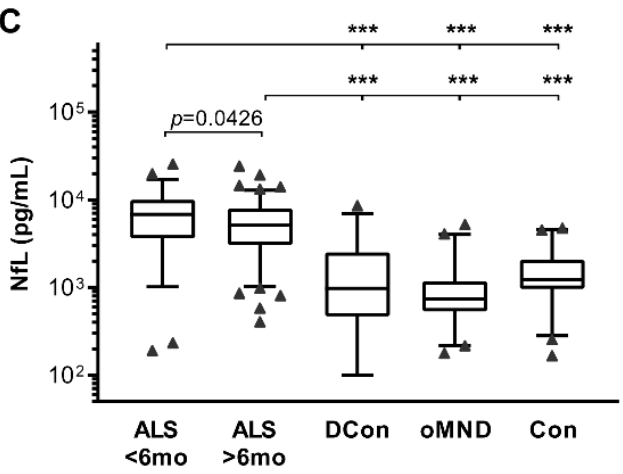

E

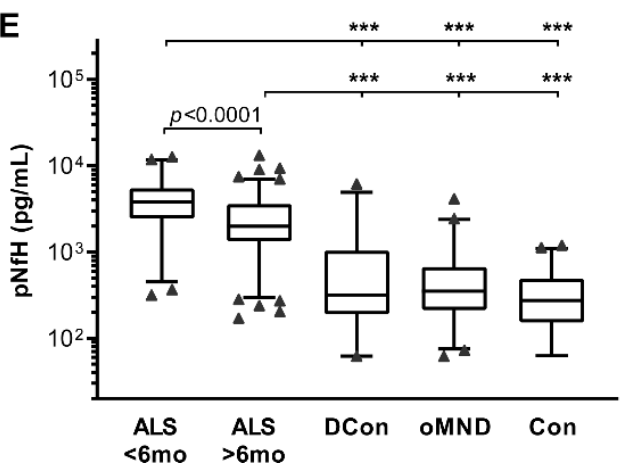

B

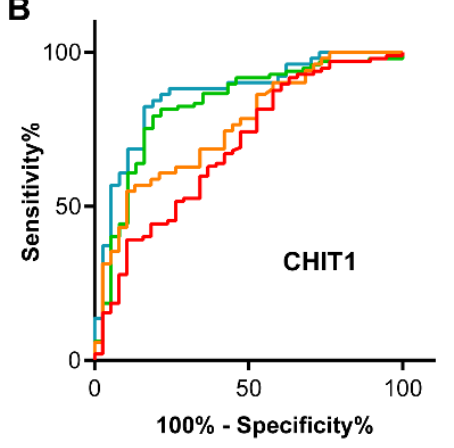

Con vs. late ALS: AUC 0.83 Sens: $79 \%$, Spez: $81 \%$

_ Con vs, early ALS AUC 0.86 Sens: $82 \%$, Spez: $84 \%$

_ DCon vs. late ALS: AUC 0.69

Sens: $88 \%$, Spez: $40 \%$

DCon vs. early ALS: AUC 0.76

Sens: $55 \%$, Spez: $89 \%$
D

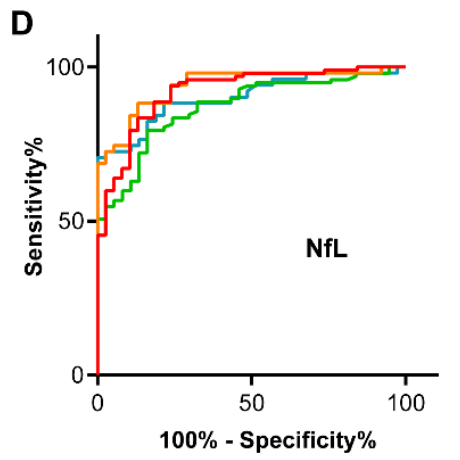

Con vs. late ALS: AUC 0.87 Sens: $88 \%$, Spez: $81 \%$

Con vs. early ALS: AUC 0.90

- Sens: $81 \%$, Spez: $86 \%$

DCon vs. late ALS: AUC 0.92 Sens: $88 \%$, Spez: $88 \%$

DCon vs. early ALS: AUC 0.94 Sens: $80 \%$, Spez: $88 \%$

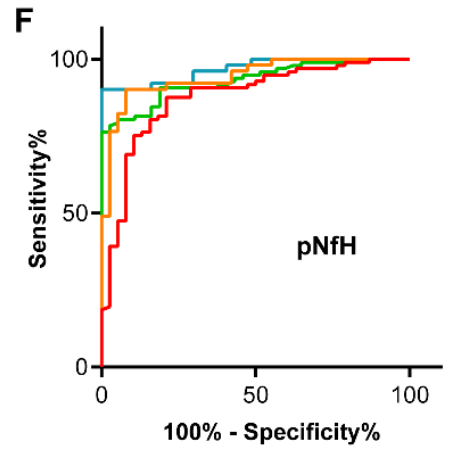

Con vs. late ALS: AUC 0.93

Sens: $85 \%$, Spez: $100 \%$

Con vs. earlyALS: AUC 0.97

Sens: $89 \%$, Spez: $100 \%$

DCon vs. late ALS: AUC 0.88 Sens: $90 \%$, Spez: $88 \%$ DCon vs. early ALS: AUC 0.94

Sens: $92 \%$, Spez: $97 \%$

Figure 2: Differential diagnostic performance of CHIT1 compared to neurofilaments

\section{for early and late symptomatic ALS.}

Boxplots of biomarker levels in the diagnostic groups and receiver operating characteristics (ROC) curves along with resulting area under the curve values, sensitivities and specificities for the discrimination of early and late symptomatic ALS subgroups from Con and DCon, respectively, for CHIT1 (A, B), NfL (C, D), and pNFH (E, F). Boxplots show median values and IQR, whiskers indicate $5 \%-95 \%$ percentile and symbols show outliers. Above the boxplots, p-values resulting from Dunn's post-hoc test for comparison of early or late ALS with the control groups, and Mann-Whitney p-values for comparison of the ALS subgroups are given. 
ALS, Amyotrophic lateral sclerosis; DCon, disease controls; oMND, other motor neuron diseases; Con, non-neurological controls.

A

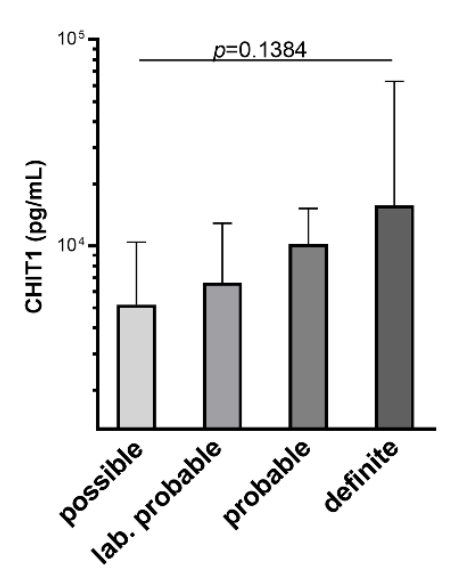

B

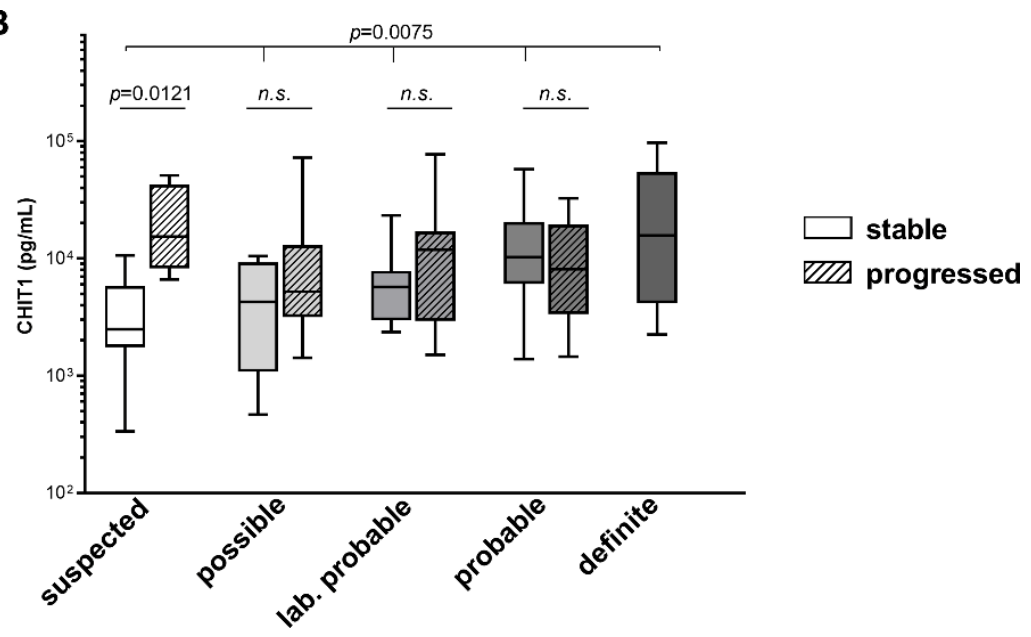

Figure 3: Comparison of CHIT1 levels with respect to clinical diagnostic category of ALS.

A: CHIT1 levels in patients diagnosed with clinical categories of ALS. B: Comparison of CHIT1 levels of ALS patients with unchanged ALS diagnostic category at follow-up and increase of certainty of ALS diagnosis at follow up. 
A

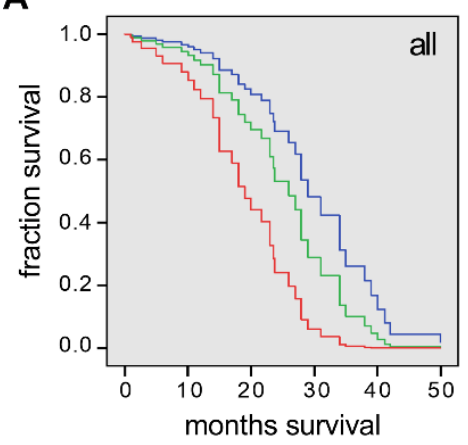

D

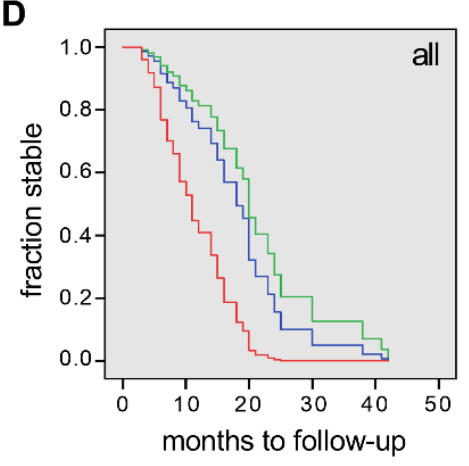

B

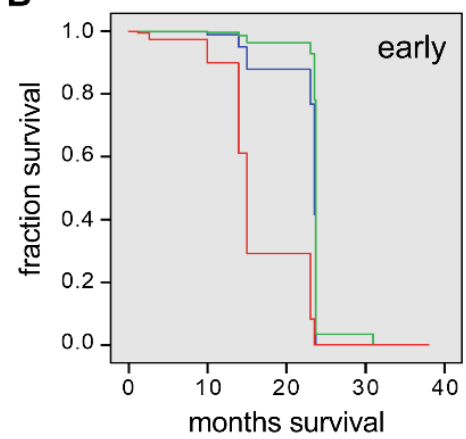

E

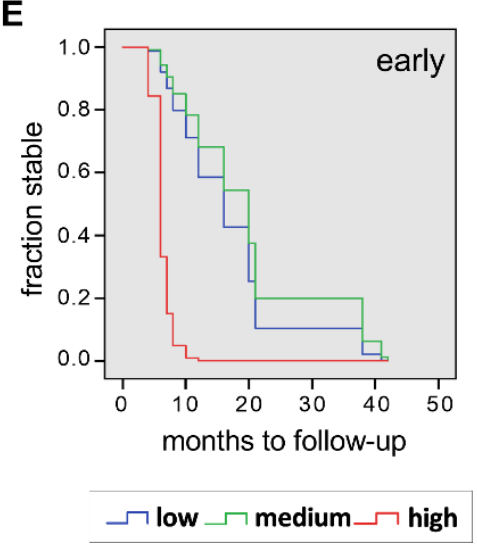

C

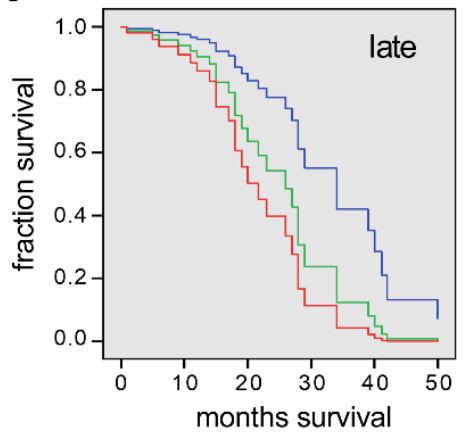

$\mathbf{F}$

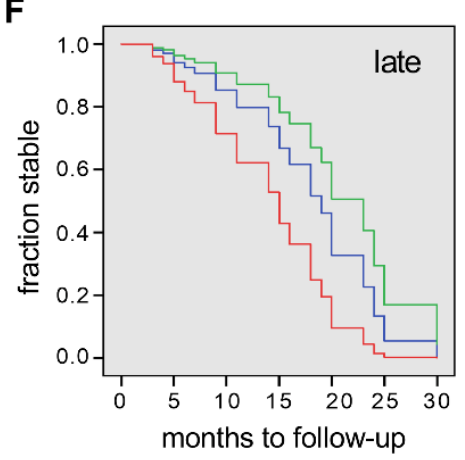

Figure 4: CHIT1 for the prediction of progression at follow-up and survival.

Shown are Kaplan-Meier plots for tertile levels (low=blue, green=medium, red=high) based on Cox proportional hazards regression analyses of CHIT1 in relation to the patients' survival time for (A) all ALS, (B) early ALS (diagnosis made within six months of disease onset), and (C) late ALS patients (diagnosis made later than six month after disease onset). As covariates, age, sex, site of onset and progression rate were implemented. Analogous plots for CHIT1 in relation to progression at follow-up regarding age, initial diagnostic category and center as covariates are shown in the lower panel for all ALS (D), early ALS (E), and late $\operatorname{ALS}(\mathbf{F})$. 